\title{
Phase Images Processing Using Parallel Programming
}

\author{
Sergei Dvoynishnikov*, Vladimir Meledin, Grigory Bakakin and Vitaly Rakhmanov \\ Kutateladze Institute of termophysics SB RAS, Russia, Novosibirsk, Lavrentieva av., 1 \\ ${ }^{*}$ Corresponding author
}

\begin{abstract}
Phase image processing algorithm with use of computational capabilities of graphics processing units (GPUs) in case of measurements of three-dimensional geometry by method of phase triangulation is offered. It is shown that the implementation of phase images decryption GPU algorithm operates more than 100 times faster than CPU version. In the case of practical implementation, performance of phase triangulation techniques will be significantly improved.
\end{abstract}

Keywords-phase triangulation; structured light; graphic processing units

\section{INTRODUCTION}

Reducing the phase images processing time in case of measurements of three-dimensional geometry by phase triangulation methods [1-2] is very important. It allows to reduce measurement time and to increase accuracy due to the statistical accumulation of experimental data. Phase images processing is reduced to solving system of transcendental equations [3]:

$$
\begin{aligned}
& I_{i}(x, y)=A(x, y)\left(1+V(x, y) \cos \left(\varphi(x, y)+\delta_{i}\right)\right), \\
& i \in 0 \ldots N-1,
\end{aligned}
$$

where $\mathrm{I}_{\mathrm{i}}(\mathrm{x}, \mathrm{y})$ - intensity distribution in the i-th image of the controlled object; $\mathrm{A}(\mathrm{x}, \mathrm{y})$ - distribution of background intensity; $\mathrm{V}(\mathrm{x}, \mathrm{y})$ - average visibility; $\varphi(\mathrm{x}, \mathrm{y})$ - sought distribution of the phase difference encoding information of range of object; $\delta_{i}-$ insertion phase shift between adjacent images of structured light; $\mathrm{N}$ - number of shifts.

In the phase steps method of data processing the phase shift of the projected harmonic signal is analyzed at each point on images of measured object regardless to the image intensity at the neighboring points. It is perspective to perform the data processing using modern parallel computing methods.

\section{METHOD DESCRIPTION}

It is possible to apply classical multiprocessor systems and multiple computer systems to effective implementation of parallel processing of phase images. However, creation of such computing systems requires considerable financial expenses. Another solution is to use other more available multiprocessor systems. These are video cards of different vendors, for example, of Nvidia and AMD companies.

Technology to improve data processing speed by using parallel computing on GPUs is actively developing in many areas of science and technology. In particular, actively develop image processing techniques to enhance computing performance [4]. Papers devoted to the processing of phase images and interferograms on GPUs, which can be used to image processing of three-dimensional scenes in methods of a phase triangulation [5] are known. These researches are focused on a narrow class of problems and can't work effectively in phase steps methods under conditions of limited dynamic range of photo detector energy, a large number of images and non-uniform reflective properties of a surface of object restricted on energy. Nevertheless, there are known works dedicated to image processing by method of phase steps for recovery three-dimensional geometry using graphic computing processors [6-7]. In these papers the approach using 3 phase images for phase determination is used. It allows to significantly restricting possibilities of error level reduction and areas of possible data applications for phase images processing methods.

We have developed and implemented a high-performance algorithm for decoding phase images with an arbitrary step-bystep shift. The method is based on the CUDA technology and steady method of phase images decoding [8].

For effective use of parallel computing it is necessary to minimize or eliminate the simultaneous access of different threads to the same data as access collisions between threads will give very strong reduction of productivity. In case of decryption of phase images an independent analysis of a phase on different pixels of the image is required.

\section{EXPERIMENTAL RESULTS}

The estimation of algorithm performance based on the sustainable processing method for phase images with arbitrary step-by-step shift implemented on the CPU and using parallel computing based on CUDA technology is performed.

The computer with the CPU Intel Core i7-4790K 4 GHz, 16 GB of RAM and NVIDIA GeForce GTX 970 graphic processor is used to analysis of algorithm operation.

Experiments for an assessment of execution time of algorithm on GPU depending on parameters of GPU computations were carried out. Figure 1 shows the processing time depending on the number of blocks. The different curves show this dependence for various amounts used threads: 32, 64, 128, 256, 512 and 1024. 


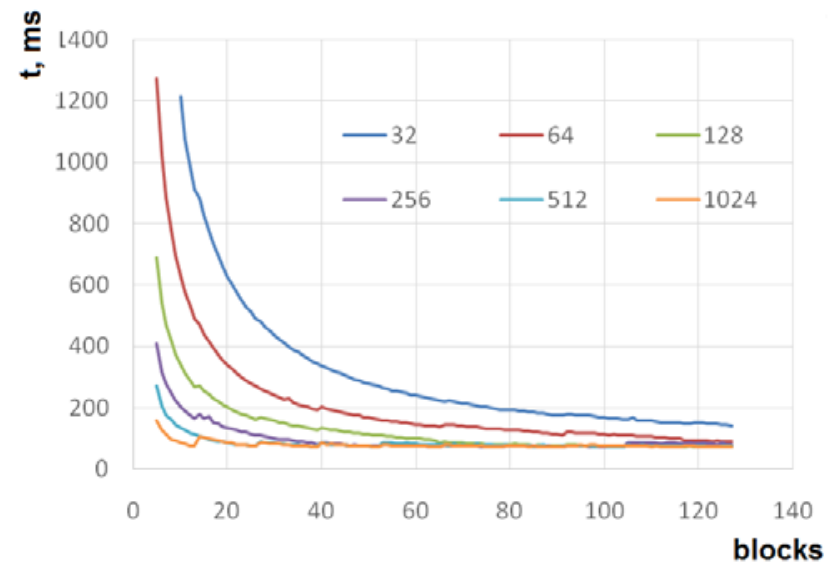

FIGURE I. DEPENDENCE OF ALGORITHM RUNTIME on the GPU the NUMBER of BLOCKS USED. THE DIFFERENT CURVES SHOW DIFFERENT QUANTITY OF threads USED IN EACH BLOCK $(32,64,128,256,512,1024)$

The graph shows that to reduce processing time it is necessary to increase the number of used blocks and threads.

Figure 2 shows dependence of GPU processing time of algorithm from the number of used blocks for the bigger number of threads. Here the configuration with quantity of threads equal to1024 in each block is provided.

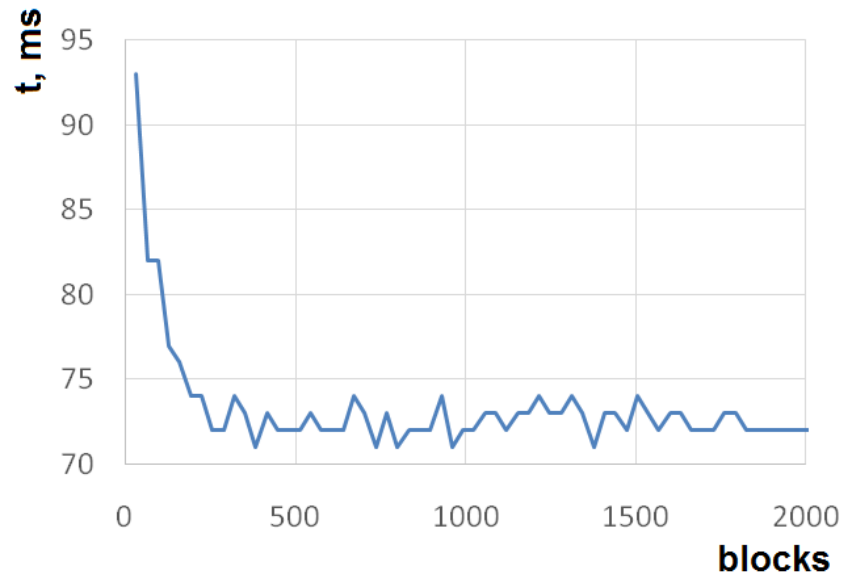

FIGURE II. DEPENDENCE OF GPU PROCESSING TIME FROM THE NUMBER OF THE USED BLOCKS

This diagram shows that in case of choice of blocks number more than 512, the processing time doesn't change.

In Figure 3 the diagram of time of data handling depending on the number of the used threads is shown. Different curves show dependence in case of different number of the used blocks: 32, 64, 128, 256, 512 and 1024.

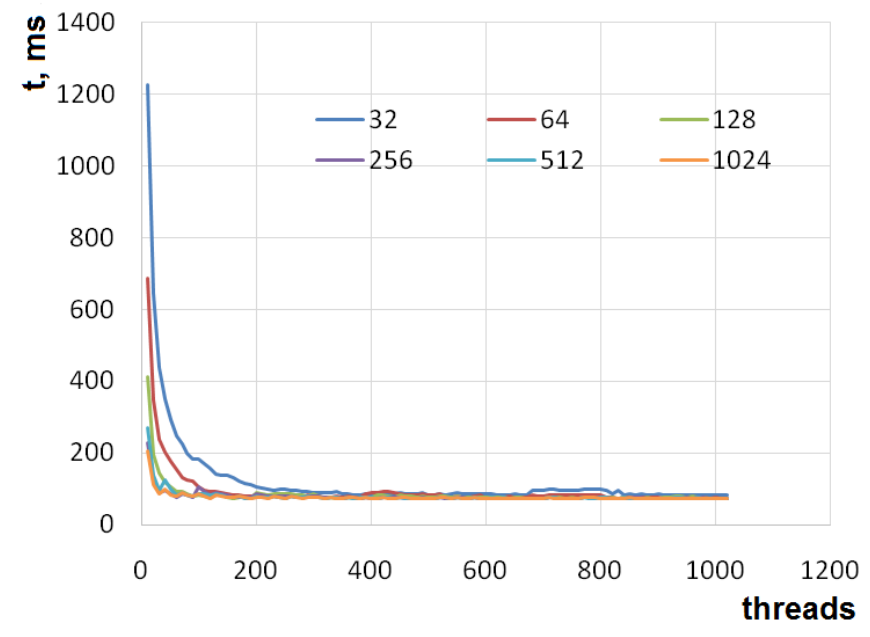

FIGURE III. THE RUNTIME OF THE ALGORITHM ON THE GPU DEPENDING ON THE NUMBER OF USED THREADS IN EACH BLOCK DIFFERENT CURVES SHOW DEPENDENCE IN CASE OF DIFFERENT NUMBER OF THE USED BLOCKS ( 32, 64, 128, 256, 512 AND 1024)

From the above graph it follows that the number of threads used effectively more than 400 .

In Figure 4 the processing time on CPU and GPU depending on the linear image size is shown. Images of the square size are used. Number of analyzed images $\mathrm{N}$ is 200 . Data processing on CUDA was executed using 512 blocks and 1024 threads in each block.

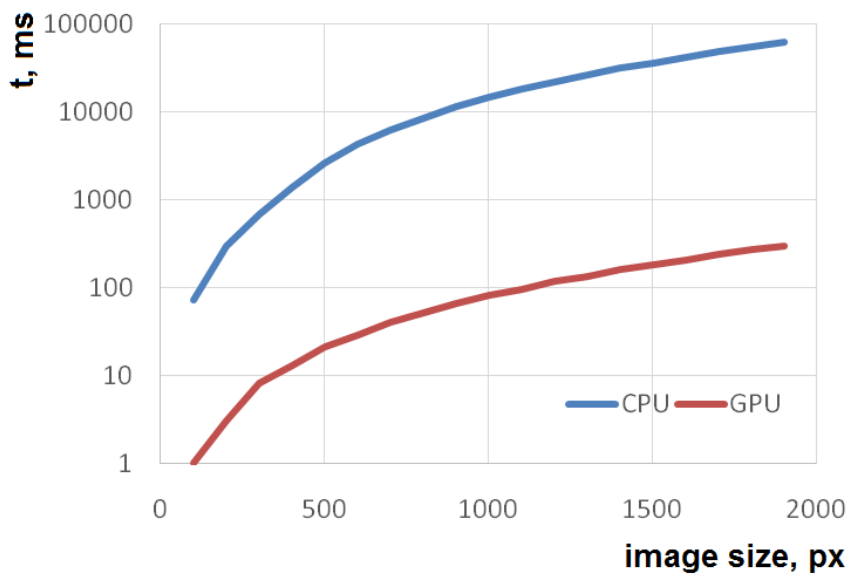

FIGURE IV. COMPARISON OF DATA PROCESSING TIME ON CPU AND GPU DEPENDING ON THE LINEAR SIZE OF THE PHASE IMAGES

As a result of researches it is established that the scoring on processing time on the GPU compared to the CPU exceeds 100 times.

\section{CONCLUTSION}

A phase image processing algorithm using the computing power of GPUs, focused on measuring the three-dimensional geometry by phase triangulation method is offered. The proposed algorithm is based on a steady method of phase images decoding with filtering the experimental data and 
harmonic regression. It is shown that implementation of the decoding algorithm of phase images on the GPU runs more than 100 times quicker than CPU implementation. The obtained results demonstrate the prospects of application of this approach for practical implementation of a phase triangulation methods.

\section{ACKNOWLEDGMENT}

Research is executed at the expense of a grant of the Russian Science Foundation (project No. 14-29-00093).

\section{REFERENCES}

[1] S. Dvoynishnikov, D. Kulikov, V. Meledin Optoelectronic method of contactless reconstruction of the surface profile of complexly shaped three-dimensional objects /Measurement Techniques 53 (6), 2010. Pp. $648-656$.

[2] S. Dvoynishnikov, V. Meledin, K. Shpolvind A method of compensation for nonlinearity of the optical radiation source-detector path in 3D measurements based on phase triangulation /Measurement Techniques 55(2), 2012. Pp. $123-129$.

[3] M. Gruber, G. Hausler: Simple, robust and accurate phase-measuring triangulation. Optik. 1992. № 3. C. $118-122$.

[4] S. Asano, T. Maruyama, and Y. Yamaguchi, "Performance comparison of FPGA, GPU and CPU in image processing," in Int. Conf. Field Programmable Logic Appl., pp. 126-131, IEEE, Prague, Czech Republic (2009).

[5] H. Kang, T. Yamaguchi, H. Yoshikawa, S. Kim, and E. Kim, "Acceleration method of computing a compensated phase-added stereogram on a graphic processing unit," Appl. Opt., 2008. Vol. 47. P. 5784-5789

[6] Karpinsky N., Hoke M., Chen V., Zhang S. High-resolution, real-time three-dimensional shape measurement on graphics processing unit // Optical Engineering, February 2014, Vol. 53(2). P. $024105-8$

[7] A. Espinosa-Romero and R. Legarda-Saenz, "GPU based real-time quadrature transform method for 3-D surface measurement and visualization // Opt. Express, 2011. Vol. 19(13). - P. 12125-12130.

[8] S.Dvoynishnikov, V.Meledin, G.Bakakin, D.Kulikov Laser Cloudy Triangulation Method for Geometrical Measurements Under the Optical Refraction Conditions / 2015 International Conference on Computer Science and Environmental Engineering, May 17-18, 2015, Beijing, China (CSEE2015) ISBN: 978-1-60595-240-6. - p.532-538. 